\title{
Clinical Features and Prognosis of Gas-forming and Non-gas-forming Pyogenic Liver Abscess: A Comparative Study
}

\section{Jia Zhang}

Xi'an Jiaotong University

\section{Yi Gao}

Xi'an Jiaotong University

\section{Zhaoqing Du}

Xi'an Jiaotong University

\section{Yifan Ren}

Xi'an Jiaotong University

\section{Jianbin Bi}

Xi'an Jiaotong University

\section{Zheng Wu}

Xi'an Jiaotong University

Yi LV

Xi'an Jiaotong University

Rongqian Wu ( $\nabla$ rwu001@mail.xjtu.edu.cn )

National Local Joint Engineering Research Center for Precision Surgery \& Regenerative Medicine https://orcid.org/0000-0003-0993-4531

\section{Research article}

Keywords: N/A

Posted Date: July 28th, 2020

DOI: https://doi.org/10.21203/rs.2.24160/v2

License: (c) (i) This work is licensed under a Creative Commons Attribution 4.0 International License. Read Full License

Version of Record: A version of this preprint was published at Surgical Infections on May 1st, 2021. See the published version at https://doi.org/10.1089/sur.2020.245. 
The authors have withdrawn this preprint from Research Square 